November 2006

\title{
Donor tricuspid annuloplasty during orthotopic heart transplantation: Long-term results of a prospective controlled study
}

Valluvan Jeevanandum

University of Chicago

Hyde Russell

University of Chicago

Paul Mather

Thomas Jefferson University

Satoshi Furukawa

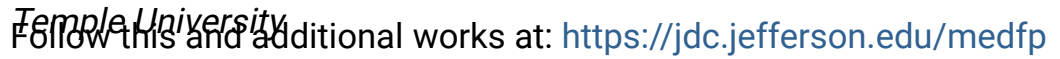

ePpaAndeksanedical Genetics Commons

universitys orkigag how access to this document benefits you

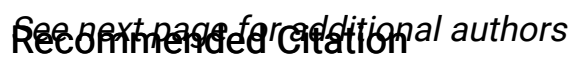

Jeevanandum, Valluvan; Russell, Hyde; Mather, Paul; Furukawa, Satoshi; Anderson, Allen; and Raman, Jaishankar, "Donor tricuspid annuloplasty during orthotopic heart transplantation: Longterm results of a prospective controlled study" (2006). Department of Medicine Faculty Papers.

Paper 14.

https://jdc.jefferson.edu/medfp/14

This Article is brought to you for free and open access by the Jefferson Digital Commons. The Jefferson Digital Commons is a service of Thomas Jefferson University's Center for Teaching and Learning (CTL). The Commons is a showcase for Jefferson books and journals, peer-reviewed scholarly publications, unique historical collections from the University archives, and teaching tools. The Jefferson Digital Commons allows researchers and interested readers anywhere in the world to learn about and keep up to date with Jefferson scholarship. This article has been accepted for inclusion in Department of Medicine Faculty Papers by an authorized administrator of the Jefferson Digital Commons. For more information, please contact: JeffersonDigitalCommons@jefferson.edu. 


\section{Authors}

Valluvan Jeevanandum, Hyde Russell, Paul Mather, Satoshi Furukawa, Allen Anderson, and Jaishankar Raman 
Title: Donor Tricuspid Annuloplasty During Orthotopic Heart Transplantation: long-term results of a prospective controlled study

Running Head: Prophylactic Donor Tricuspid Annuloplasty

Authors: Valluvan Jeevanandam, $\mathrm{MD}^{1}$, Hyde Russell, $\mathrm{MD}^{1}$, Paul Mather, $\mathrm{MD}^{3}$, Satoshi Furukawa, $\mathrm{MD}^{2}$, Allen Anderson, MD¹, Jaishankar Raman, MD, PhD ${ }^{1}$

Institution and Affiliations: ' University of Chicago, 5841 South Maryland Avenue, MC5040, Chicago, Illinois 60637, Departments of Surgery and Medicine

${ }^{2}$ Temple University, 3401 N. Broad Street, Philadelphia, Pennsylvania 19140

Department of Surgery

${ }^{3}$ Thomas Jefferson University, 925 Chestnut St. Philadelphia, Pennsylvania 19107

Departments of Medicine

Meeting Presentation: The Society of Thoracic Surgeons 42th Annual Meeting

Chicago, IL: January 30 - February 1, 2004

Key Words: Cardiac transplantation, Tricuspid valve surgery, Regurgitation

Word Count: 4,486

Corresponding Author: Valluvan Jeevanandam, MD, Section of Cardiac \& Thoracic Surgery, University of Chicago, 5841 South Maryland Avenue, MC5040, Chicago, Illinois 60637;

Telephone: 773.702.2500; Fax: 773.834.9114; email: jeevan@uchicago.edu.

Jeevanandam, et al. Page 1 of 24 


\section{Abstract:}

Background: Development of tricuspid regurgitation (TR) after orthotopic heart transplantation $(\mathrm{OHT})$ can cause heart failure along with renal and hepatic impairment and portends a poor prognosis. If TR causes significant symptoms, tricuspid valve repair or replacement is often required. This study was designed to study the effects of prophylactic tricuspid valve annuloplasty (TVA) during OHT on long-term survival, renal function and amount of TR. Methods: Between 4/97 and 3/98, 60 patients (age 18-70, 22 females) randomly received either OHT ( $n=30$, group STD) or OHT with DeVega TVA $(n=30$, group TVA). TVA was performed on the donor heart before implantation using pledgeted 2-0 prolene and sized to an annulus of $29 \mathrm{~mm}$. Echocardiographic parameters, lab values and hemodynamics were obtained prospectively and reviewed by an independent data analyst. Results: Follow-up of patients up to December 2003 was complete. Although, there was a peri-operative mortality advantage in group TVA, there was no difference in long-term survival. However, at the end of the study period there was a statistical difference (groups STD vs, TVA; $p<0.05$ ) with regard to cardiac mortality (7/30 vs. $3 / 30)$, average amount of $\operatorname{TR}(1.5 \pm 1.3$ vs. $0.5 \pm 0.4)$, percentage of patients with $\geq 2+\operatorname{TR}(34 \%$ vs. $0 \%)$, serum creatinine $(2.9 \pm 2.0$ vs $1.8 \pm 0.7)$ and difference in serum creatinine over baseline $(2.0 \pm 2.1$ vs $0.7 \pm 0.8)$. Conclusion: Prophylactic DeVega TVA of the donor heart is durable and decreases the incidence of cardiac related mortality and postOHT TR. In addition, there is improved protection of renal function. Considering the ease and safety of TVA and its advantages, it should be performed as a routine adjunct to OHT.

Jeevanandam, et al. Page 2 of 24 


\section{Introduction:}

Orthotopic heart transplantation $(\mathrm{OHT})$ is often associated with the development of tricuspid regurgitation (TR) [1,2] with the reported incidence varying from 47 to $98 \%[3,4,5]$. The TR can be related structural or functional factors. The standard biatrial technique of $\mathrm{OHT}(\mathrm{sOHT})$ is associated with more TR than the bicaval (bOHT) technique. This difference is attributed to alteration in the geometry of the tricuspid valve and right atrium related to the technique of right atrial anastomosis. Other possible causes of TR include: 1) allograft dysfunction with right ventricular dilatation due to poor preservation, reperfusion injury, donor factors, or rejection, 2) pulmonary hypertension, 3) severe donor recipient size mismatch, and 4) structural damage occurring during endomyocardial biopsy $[6,7,8,9,10]$.

Moderate and greater grades of TR are associated with right sided heart failure symptoms, renal and hepatic dysfunction, and decreased long-term survival $[11,12]$. It remains unclear whether the TR is a reflection of poor allograft function or a primary abnormality, but once present, repair or replacement is often indicated. Renal dysfunction is also a long-term consequence of $\mathrm{OHT}$. This is most commonly attributed to the calcineurin inhibitors and may also result from TR $(13,14,11$, ISHLT registry data).

The DeVega type of tricuspid annuloplasty using a suture has been used to treat TR. It is simple, quick, inexpensive and durable $[15,16]$. We have previously reported short-term results on a population of randomized and prospectively studied patients, one group who received prophylactic DeVega TVA to prevent TR after OHT(17). We demonstrated less TR, improved peri-operative heart function and survival. This paper reports on the long-term (six year) followup of those patients and compares them with regard to survival, amount of TR, renal function and hemodynamics.

Jeevanandam, et al. Page 3 of 24 


\section{Methods}

\section{Study Design:}

The study design has previously been described (17). Of the 87 patients receiving heart transplants at Temple University Hospital between 4/1997 to 4/1998, 60 patients met selection criteria for this comparison. Institutional Review Board permission was received (3/1997) before studying these patients. Twenty-seven patients were excluded because of: 1) multiple organ transplantation, 2) re-transplantation, 3) donor/recipient weight ratio $<0.5,4$ ) pulmonary vascular resistance $(P V R)>4$ woods units, 5) greater than mild TR documented on donor echocardiography, 6) donor ejection fraction <35\%, 7) donor coronary artery disease requiring revascularization, 8) donors requiring two inotropes or dopamine at $>12 \mu \mathrm{g} / \mathrm{kg} / \mathrm{min}$. In accordance with our practice at that time, these patients that were deemed higher risk as evidenced by one or more of the listed factors, routinely received TVA before the transplant procedure.

Two surgeons performed the procurement and implantation during this time period, and the procedures were evenly distributed among them. TVA was performed during preparation of the donor heart. In order to insure consistency and standardization, only one of the surgeons performed the TVA. By strictly adhering to an evenly distributed procurement call schedule, randomization was achieved. Patients were not included in the comparison if unforeseen circumstances caused deviation from the on-call schedule. In the majority of cases, implantation was a combined effort of the two surgeons. The patients received a OHT (group STD) or OHT with TVA (group TVA). Data were obtained prospectively and data analysts were blinded to the assignment of patient groups. Data analyzed included: operative and donor information, survival, amount of TR, hemodynamics, serum BUN and creatinine.

\section{Procedure:}

University of Wisconsin (Barr Laboratories, Pomona, NY) solution was used for cardioplegia and preservation solution. The recipient cardiectomy and recipient-donor anastomoses were 
done using the bicaval technique described by Sarsam, et al. [18] and others [19]. All patients received a baseline inotropic regiment of T3 $(0.08 \mu \mathrm{g} / \mathrm{kg}$ bolus, infusion $0.8 \mu \mathrm{g} / \mathrm{kg}$ for 3 hours), $5 \mu \mathrm{g} / \mathrm{kg} / \mathrm{min}$ of dobutamine and $3 \mu \mathrm{g} / \mathrm{kg} / \mathrm{min}$ of dopamine. Any increase above baseline was considered as elevated inotropic requirement. Patients were considered weanable from CPB if they could sustain a mean systemic blood pressure $>70 \mathrm{mmHg}$ with a CVP of $<15 \mathrm{mmHg}$ and a cardiac index $(\mathrm{Cl})>2.1 \mathrm{~L} / \mathrm{min} / \mathrm{M}^{2}$ with a combination of inotropic and IABP support.

Group TVA patients received a DeVega type annuloplasty on the donor graft prior to implantation. The tricuspid valve was visualized through the inferior caval or right atrial opening and a double layer of pledgeted 2-0 polypropylene was used to stabilize the annulus. The annulus was sized to a $29 \mathrm{~mm}$ dilator. If the annulus was less than $29 \mathrm{~mm}$, it was stabilized to a size just a little smaller than the native annulus.

\section{Management:}

All patients received triple-drug immunnosuppression therapy with cyclosporine (CyA) (3.5-5.0 $\mathrm{mg} / \mathrm{kg} / \mathrm{day})$, azathioprine (1.5-2.5 mg/kg/day) and steroids. Induction therapy was not routinely used. Patients either had the CyA switched to tacrolimus or had mycophenolate mofetill substituted for azathioprine for repeated rejection. CyA therapy was adjusted to maintain serum trough levels of 250 to $300 \mathrm{ng} / \mathrm{ml}$ during the first year after HT. Azathioprine dose was adjusted to maintain white blood cell counts $>4,000 / \mathrm{ml}$. Steroid dose was tapered gradually after the peri-transplant period to $0.05 \mathrm{mg} / \mathrm{kg}$ within six months after surgery. Acute rejection $(>1 \mathrm{~b}$ or with hemodynamic compromise) was treated with a steroid bolus. Cytolytic therapy was added for repeated rejections or significant hemodynamic compromise. Endomyocardial biopsy was performed using the standard Stanford technique with either a Caves-Shultz or disposable bioptome[20]. Biopsy-proven rejection was graded using the modified Billingham system adopted by the International Society for Heart and Lung Transplantation [21]. 


\section{Echocardiographic assessment of TR}

Patients routinely underwent intra-operative transesophageal echocardiography (TEE), and then transthoracic (TTE) studies at one week, one month, and then annually. As part of the echocardiographic studes, patients received two-dimensional and continuous-wave Doppler evaluation with color flow mapping. The tricuspid valve was examined in the parasternal shortaxis and apical four-chamber views. Tricuspid regurgitation was graded by qualitative echocardiographic assessment according to the ratio of the regurgitant jet area to the right atrial area, as described previously [18]. Grades of TR included: $0,1+$ (jet to area ratio $<10 \%$,trace), $2+$ (ratio 10-24\%, mild), 3+ (ratio 25-49\%, moderate), 4+ (ratio $>50 \%$, severe). Multiple views were obtained and the regurgitation graded based upon the most abnormal views.

Hemodynamic Assessment

Intra-operative pulmonary artery monitoring with a Swan-Ganz catheter was performed in every patient. Right heart catheterization was routinely performed with biopsy at one week, one month, and annually, or if dictated by clinical condition. Intra-cardiac pressures were recorded at the levels of the right atrium, right ventricular body, and pulmonary artery. Thermodiluton cardiac outputs were also obtained.

\section{Statistics}

All numerical results are reported as mean \pm standard deviation. A p value of less than 0.05 was considered to be significant. Statistical analysis was performed using SPSS v.11 (SPSS Inc, Chicago, IL). Fisher's exact test was applied for associations of categorical variables, and the Wilcoxon rank sum test was used to compare means of continuous variables. Survival analysis was performed using the log rank statistic.

Jeevanandam, et al. Page 6 of 24 


\section{Results:}

General Characteristics: There were 30 patients in each group. Recipient and donor demographics are presented in tables 1 and 2 respectively. Pre-transplant pulmonary artery pressures were similar in both groups (STD vs. TVA, $p=n s$ : mPAP 28.6 \pm 9.2 vs. 29.3 \pm 8.9 ; PCWP 23.2 \pm 8.9 vs. $23.8 \pm 9.0$; PVR Woods units $2.3 \pm 0.9$ vs. $2.1 \pm 1.1$ ). The two surgeons who performed the procedures were randomly distributed among the procurement and implantation groups, based on the call schedule. Both groups followed the same routine myocardial biopsy schedule. The average number of biopsies for the first year in survivors of both groups was 15.2. After the first year, patients received routine biopsies bi-annually unless clinically indicated. None of the patients required a permanent pacemaker. There was no incidence of tricuspid stenosis.

Tricuspid regurgitation: The average amount of TR in each group was stable during the study period. TEE evaluation of patients after weaning from CPB and stabilization revealed more TR in group STD (table 3). The difference in average TR persisted at one and six years. Moreover, the standard group had a greater incidence of $\geq 2+T R$. Subsequently, there was a progressive increase in the number of patients with grade $\geq 3+$ TR in group STD. Group TVA had a stable distribution of TR severity. No patients in group TVA developed significant TR $(\geq 2+)$. By six years, three patients in group STD demonstrated clinical signs of TR with peripheral edema and increased diuretic requirements. Two required a tricuspid valve procedure. There is a significant correlation between $T R \geq 2+$ and mortality as well as $T R$ and increase in creatinine (figures 1 and 2).

Hemodynamics: The hemodynamic values (table 4) at one and six years were similar.

Renal function: The BUN was similar in the two groups. The baseline creatinine in group TVA seems higher in group STD $(1.34 \pm 0.67$ vs $1.02 \pm 0.29)$, but it fails to attain significance. The serum creatinine increased in both groups after transplant, and continued to increase in group STD while stabilizing in group TVA. Serum creatinine and the difference in creatinine from 
baseline were significantly higher in group STD at 6 years (table 5). In the overall population of patients, there is a correlation between creatinine $\geq 2+$ and mortality (figure. 3 )

Morbidity and Mortality: Overall survival among both groups was similar with 8 patients expiring in both groups. Group STD had a higher intra-operative mortality (4/30 vs $0 / 30)$. Three deaths were due primarily to right heart failure and one to an acute rejection. Although, the patients eventually died of multiple system organ failure, the root cause was low cardiac output. By the first and sixth years, survival was similar between groups (table 7). Of note, 7/8 deaths in Group STD were cardiac in origin. One patient died of a malignancy. In group TVA, there were 3 cardiac deaths, two perforated visceral organs and 3 infections. There were statistically more cardiac related deaths in group STD $(p=0.03)$. The average number of treated rejection episodes per patient was similar between groups.

\section{Comment:}

Tricuspid regurgitation frequently develops following OHT and can be progressive [11]. Variability exists between the common techniques for measuring TR [5]. The color doppler quantification method is the one used in this study and is the technique that is most widely accepted(4). Using this technique, Huddleston et al. found that $21 \%$ had greater than moderate TR [3]. Williams et al. found a $32 \%$ prevalence of moderate to severe TR following heart transplantation [23]. Aziz et al. found that $36 \%$ of patients developed greater than moderate TR after OHT [11]. In the largest study to date, the Stanford group analyzed 336 patients and found $34 \%$ had greater than moderate TR. In addition, the studies that follow patients over a period of time demonstrate the progressive nature of this abnormality. For instance, in the Stanford experience, the incidence of severe TR increases in survivors from $7.8 \%$ at five years to $14.2 \%$ at ten years [5].

TR can become a serious condition. If the TR progresses past moderate, a majority of patients can develop peripheral edema, ascites and renal dysfunction. Furthermore, the long 
term survival in patients with moderate TR is decreased when compared to patients with less than mild TR $[11,5]$. In a study analyzing intra-operative echocardiograms, immediate significant TR was associated with a poor long term survival. The authors suggest a role for concomitant TVA at the time of transplant [12].

When severe TR is present, repair with an annuloplasty or replacement has been reported $[2,24]$. DeVega tricuspid annuloplasty is an inexpensive, quick and stable method to reduce a dilated annulus. It has been primarily used to treat functional TR caused by elevated left sided pressures with excellent long term results $[15,16]$. This study defined the role of prophylactic TVA. Whether the TVA is best accomplished by a DeVega technique or by a ring is based on surgeon preference. Stabilization with an annuloplasty ring is preferable in patients with severe TR and dilatation of the annulus. However, in this study, none of the patients had an abnormally dilated annulus. The DeVega annuloplasty was performed to maintain the normal size of the annulus. This was clearly accomplished in the study as there was no progression or development of new TR in group TVA. The technique employed in the performance of the DeVega annuloplasty is important: careful and closely spaced sutures with a double pledgeted repair is critical in maintaining the integrity of the annulus. We have used an annuloplasty ring in other patients but have found the bulky prosthetic material to be a hindrance for biopsies, which makes these procedures time consuming and expensive. Complications of the TVA are uncommon, and are primarily related to injury of the conduction system. It is also important not to create stenosis by reducing the annulus excessively. Tricuspid stenosis or heart block did not occur in any patient.

TR following OHT is often a complication of the endomyocardial biopsy procedure $[23,25,26,27]$. Many studies correlate the presence of flail leaflets and torn chordae with severe TR. In fact, almost all reported cases of TV repair or replacement after OHT have demonstrated mechanical disruption of the TV. There is no reason to believe that a TVA would prevent mechanical disruption of the valve mechanism. However, since the annulus is decreased, TR 
from minor structural damage can be attenuated. Two patients in group TVA had biopsy proven injury to the chordal structures. Subsequent echocardiograms confirmed ruptured chorde. However, the TR did not increase above a mild degree.

Other causes of TR have a functional basis. The presence of pulmonary hypertension may cause right ventricular and annular dilatation causing TR. One study demonstrated a correlation between pulmonary hypertension and TR. However, several other studies have shown no correlation between pre- or post-operative recipient pulmonary hypertension and development of severe TR $[23,7,9]$. Pulmonary hypertension by itself with a normal RV appears to be well tolerated; superimposition of pulmonary injury or RV dysfunction may contribute to a process leading to development of TR. RV dysfunction can be caused in the intra-operative period by preservation or reperfusion injury, air embolus, donor risk factors, or accelerated rejection. The short term results of this study (17) demonstrated improved peri-operative survival, especially when these stresses were imposed on a donor organ. Long-term, there was a decrease in cardiac associated mortality. If a cardiac related stress is superimposed on a transplant patient, the TVA allows of maintenance of myocardial efficiency, lower CVP and perhaps enough restoration of perfusion to allow these patients to survive. However, $\mathrm{OHT}$ is associated with many factors that can cause death, such as infection and malignancy. Hence, despite being superior with regard to intra-operative and cardiac survival, the overall mortality in both groups was similar.

We exclusively used a bicaval technique for $\mathrm{OHT}$. There is ample evidence that bicaval $\mathrm{OHT}$ is superior to standard biatrial $\mathrm{OHT}$ in preventing TR. This is attributed to elimination of the $\mathrm{RA} / \mathrm{RV}$ relationship caused by the biatrial implantation technique. In a study of 249 patients, Aziz et al. report a $19.9 \%$ incidence of severe TR in the bOHT group as opposed to $51 \%$ for the sOHT group[11]. Since there was evidence that bicaval OHT decreases TR and improves sinus node function $[28,29]$, we used this technique for all patients. If the standard OHT technique had been utilized, the prophylactic TVA might theoretically be of additional benefit. 
We tested the hypothesis that prophylactic TVA, done before OHT, could impact the incidence of TR, the need for further TV procedures and patient outcomes. This was a single center study and was, therefore, small. Two surgeons were responsible for all the transplant procedures. It was important not to alter normal practice, while standardizing the TVA and removing surgeon variability as a confounding factor in the comparison. Therefore only one surgeon performed the TVA. Randomization was ensured by strictly following an evenly distributed on-call schedule. Implantation was also evenly distributed among the two surgeons. Of the sixty implantation procedures, 45 were a combined effort of the surgeons. There was no correlation between surgeon and intra-operative complications. This study could not be blinded to the surgeon. Data analysts were blinded as much as possible, but in a majority of cases, the pledgets of the TVA were evident on TEE or TTE as an echo density.

The pre-transplant status of recipients and donors were similar in both groups. The significant differences at long-term follow-up included prevention of significant TR and renal protection. The amount of TR was low, as would be expected from the bicaval technique, but was still different among the groups. In the early peri-transplant period, group STD had a mean of 1.1 vs group TVA .33 TR. This is represented as a mean of only mild TR, but there was increased mortality in the patients who developed greater than moderate TR (17). Group TVA patients had only trivial TR; even the patients with myocardial dysfunction developed only mild TR. The difference in average TR continues to be present at one and six years of follow-up. Furthermore, in group STD, the percentage of patients with $\geq 2+$ TR remains stable at $\sim 30 \%$ throughout the study period. The incidence of moderate/severe TR $(>3+)$ in group STD is $7.6 \%$ at one year and compares favorably to other reports using bOHT techniques. At six years, the severe TR group increases to $22.7 \%$ of the survivors. At one and six years, there were no patients with greater than mild TR in group TVA. Three patients in group STD developed clinical symptoms from central venous hypertension. Two required operative correction. It is evident from other reports that TR is progressive and can lead to worsening right heart failure 
symptoms. That is consistent with the STD group in this study. Furthermore, follow-up of patients in this study demonstrates that a TVA prevents development and progression of TR.

The other parameter which we studied was renal function, since there are data to correlate renal dysfunction with worsening TR[11,12,13]. Increased CVP in patients with TR contributes to increased renal venous pressure, which has been shown to be responsible for decreased renal artery flow. This in turn increases plasma rennin activity, causes urinary protein leak, and decreases glomerular filtration rate. This explains the deterioration in renal function in patients with significant TR [11]. In this study, patients' serum creatinine progressively increased during the first year in both groups. However, by 6 years, there is stabilization of renal function in group TVA and the different average levels of serum creatinine and the difference from baseline from group STD are significant. Furthermore, there is significant correlation between development of increased creatinine and mortality. Calceneurin inhibitors can contribute to renal dysfunction and that effect is present in both groups. However, the development of TR is an added insult and correlates with renal dysfunction. This effect is evident only after long-term follow-up and the development of moderate TR with deterioration in renal function.

There were several limitations for this study. Since it was a single center study, it was relatively small. The study could not be properly blinded due to the performance of a surgical procedure in the OR and the visibility of the TVA on echocardiography. One could argue that both surgeons should have performed the TVA. We felt that since this was a small study, it was important to remove any variability in the TVA technique and standardize the procedure. If this was a larger study, involvement of other surgeons would have decreased some of the variability. The study was also conducted prior to approved use of nitric oxide and expanded use of mechanical devices to support the right ventricle. It is possible that the mortality benefit might have decreased with routine early use of a right ventricular assist device such as the Abiomed BVS 5000 (Abiomed, Danvers, MA). 
In summary, this is the first long-term report of using prophylactic TVA to decrease TR after OHT. Previous reports using this patient population demonstrated survival benefit to TVA in the immediate post-operative period. This is the period of time when the heart is maximally stressed and the patients are vulnerable to the effects of a low perfusion state. This cardiac protective effect is present long-term as demonstrated by less cardiac related deaths. However, the overall survival is similar. The study also demonstrates significant reduction in the mean severity and number of patients with significant TR. This prevention of TR has important long term beneficial effect as the presence of moderate TR is progressive and associate with a poor prognosis. Since there is correlation between development of TR and renal dysfunction, the TVA helps preserve renal function. Prophylactic DeVega TVA is inexpensive, quick to perform, durable at one year, and offers a survival advantage in the immediate post operative period. Long-term, there is better preservation of renal function and prevention of TR. Since TVA is beneficial, it should be routinely considered during preparation of a donor heart for transplantation.

\section{Acknowledgments:}

We thank Karen Bradfield and Ursula Williams for their assistance in preparation of this manuscript.

Jeevanandam, et al. Page 13 of 24 


\section{REFERENCES}

1. Sahar G, Stamler A, Erez E, et al. Etiological Factors Influencing the Development of Atrioventricular Valve Incompetence After Heart Transplantation. Transplantation Proceedings 1997;29:2675-2676.

2. Ichikawa S, Takeuchi Y, Suda Y, et al. Tricuspid Valve Replacement After Cardiac Transplantation. JJTCVS 2000;48:659-662.

3. Huddleston C, Rosenbloom M, Goldstein J, et al. Biopsy-induced tricuspid regurgitation after cardiac transplantation. Ann Thorac Surg 1994;57;(4):832-6.

4. Lewen M, Bryg R, Miller L, et al. Tricuspid regurgitation by Doppler echocardiography after orthotopic cardiac transplantation. Am J Cardiol 1987;59(15):1371-4.

5. Chan M, Giannetti N, Kornbluth M, et al. Severe Tricuspid Regurgitation After Heart Transplantation. J Heart Lung Transplant 2001;20:709-717.

6. Bainbridge A, Cave M, Roberts M. A prospective randomized trial of complete atrioventricular transplantation versus ventricular transplantation with atrioplasty. J Heart Lung Transplant 1999;18:407-13.

7. Aziz T, Burgess M, Rahman A, et al. Risk factors for tricuspid valve regurgitation after orthotopic heart transplantation. Ann Thorac Surg 1999;68(4):1247-51.

Jeevanandam, et al. Page 14 of 24 
8. De Simone R, Lange R, Sack F, et al. Atrioventricular valve insufficiency and atrial geometry after orthotopic heart transplantation. Ann Thorac Surg 1995;60(6):1686-3.

9. Leyh R, Jahnke A, Kraatz E, et al. Cardiovascular dynamics and dimensions after bicaval and standard cardiac transplantation. Ann Thorac Surg 1995;59(6):1495-1500.

10. Deleuze P, Benvenuti C, Mazzucitelli J, et al. Orthotopic cardiac transplantation with direct caval anastomosis: is it the optimal procedure? J Thorac Cardiovasc Surg 1995;109(4):731-7.

11. Aziz T, Saad R, Burgess M, et al. Clinical Significance of Tricuspid Valve Dysfunction After Orthotopic Heart Transplantation. J Heart Lung Transplant 2002;21:1101-1108.

12. Anderson CA, Stanton SK, Leacche M, et al. Severity of intraoperative tricuspid regurgitation predicts poor late survival following cardiac transplantation. Ann Thorac Surg $2004 ; 78$ :163543

13. Cipullo R, Finger MA, Ponce F, et al. Renal failure as a determinant of mortality after cardiac transplantation. Transplantation Proceedings 2004;36:989-990

14. Vossler MR, Ni H, Toy W, et al. Pre-operative renal function predicts development of chronic renal insufficiency after orthotopic heart transplantation. J Heart Lung Transplant $2002 ; 21: 874-881$

15. Morishita A, Kitamura M, Noji S, et al. Long-term results after De Vega's tricuspid annuloplasty. J Cardiovasc Surg(Torino) 2002;43(6):773-7. 
16. Kuwaki K, Morishita K, Tsukamoto M, et al. Tricuspid valve surgery for functional tricuspid valve regurgitation associated with left-sided valvular disease. Eur J Cardiathorac Surg $2001 ; 20(3): 577-82$

17. Jeevanandam $\mathrm{V}$, Russell $\mathrm{H}$, Mather $\mathrm{P}$, et al. A one-year comparison of prophylactic donor tricuspid annuloplasty in heart transplantation. Ann Thorac Surg 2004 ;78(3) :759-66

18. Sarsam R, Campbell C, Yonan N, et al. An alternative surgical technique in orthotopic cardiac transplantation. J Cardiovasc Surg 1993;8:344-9.

19. Aziz T, Burgess M, El-Gamel A, et al. Bicaval and standard techniques in orthotopic heart transplantation: medium term experience in cardiac performance and survival. J Thorac Cardiovas Surg 1999;118:115-22.

20. Caves $\mathrm{P}$, Stinson E, Billingham M, et al. Percutaneous transvenous endomyocardial biopsy in human heart recipients: experience with a new technique. Ann Thorac Surg 1973;16(4):325-36.

21. Billingham M, Cary N, Hammond M, et al. A working formulation for standardization of nomenclature in the diagnosis of heart and lung rejection: Heart Rejection Study Group. The International Society for Heart Transplantation. J Heart Transplant 1990;9(6):587-93.

22. Mugge A, Daniel W, Herrmann G, et al. Quantification of tricuspid regurgitation by Doppler color flow mapping after cardiac transplantation. Am J Cardiol 1990;66(10):884-7.

23. Williams M, Lee M, Disalvo $\mathrm{T}$, et al. Biopsy-induced flail tricuspid leaflet and tricuspid regurgitation following orthotopic cardiac transplantation. Am J Cardiol 1996;77(15):1339-44. 
24. Crumbley A III, Van Bakel A. Tricuspid valve repair for biopsy-induced regurgitation after cardiac transplantation. Ann Thorac Surg 1994;58(4):1156-60.

25. Braverman A, Coplen S, Mudge G, et al. Ruptured chordae tendineae of the tricuspid valve as a complication of endomyocardial biopsy in heart transplant patients. Am J Cardiol 1990;66(1):111-3.

26. Hausen B, Albes J, Rohde R, et al. Tricuspid valve regurgitation attributable to endomyocardial biopsies and rejection in heart transplantation. Ann Thorac Surg 1995;59(5):1134-40.

27. Tucker P II, Jin B, Gaos C, et al. Flail tricuspid leaflet after multiple biopsies following orthotopic heart transplantation: echocardiographic and hemodynamic correlation. J Heart Lung Transplant 1994;13(3):466-72 .

28. Brandt M, Harringer W, Hirt S, et al. Influence of bicaval anastamoses on late occurrence of atrial arrhythmia after heart transplantation. Ann Thorac Surg 1997;64:70

29. Rothman S, Jeevanandam V, Combs W, et al. Eliminating bradyarrhythmias after orthotopic heart transplantation. Circulation 1996;94:278-82 
Table 1 - Recipient Demographics

\begin{tabular}{ccccccccc} 
Group & Age & M/F & Height $(\mathrm{cm})$ & Weight $(\mathrm{kg})$ & Cause & UNOS & Mech & Reop \\
\hline STD & $53.1 \pm 12.2$ & $18 / 12$ & $169.2 \pm 12.7$ & $80 \pm 12.7$ & Ish 13 & I 27 & 5 & 12 \\
& & & & & Idio 16 & II 3 & & \\
& & & & & PP 1 & &
\end{tabular}

$\begin{array}{lllllllll}\text { TVA } & 51.1 \pm 11.7 & 20 / 10 & 172.2 \pm 10.7 & 79.5 \pm 18.7 & \text { Ish } 13 & \text { | } 26 & 5 & 13\end{array}$ Idio 17 II 4

PP 0

$\begin{array}{llllll}\text { ns } & \text { ns } & \text { ns } & \text { ns } & \text { ns ns }\end{array}$

$\mathrm{M} / \mathrm{F}:$ male/female

Cause: Ish - ischemic; Idio - idiopathic; PP - postpartum

UNOS: United Network of Organ Sharing listing status

Mech: mechanical assistance including LVAD or IABP

Reop: previous sternotomy

Jeevanandam, et al. Page 18 of 24 
Table 2 - Donor Demographics

\begin{tabular}{cccccc} 
Group & Age & M/F & Height $(\mathrm{cm})$ & Wt(kg) & D/R ratio \\
\hline STD & $26 \pm 13.1$ & $22 / 8$ & $161 \pm 35.9$ & $65 \pm 19.5$ & $0.8 \pm 0.2$ \\
& & & & & \\
TVA & $26.9 \pm 12.6$ & $19 / 11$ & $167.5 \pm 16.2$ & $67.5 \pm 19.5$ & $0.8 \pm 0.2$ \\
& ns & ns & ns & ns & ns
\end{tabular}

D/R ratio: ratio of donor/recipient weight 
Table 3 - Distribution of the severity TR

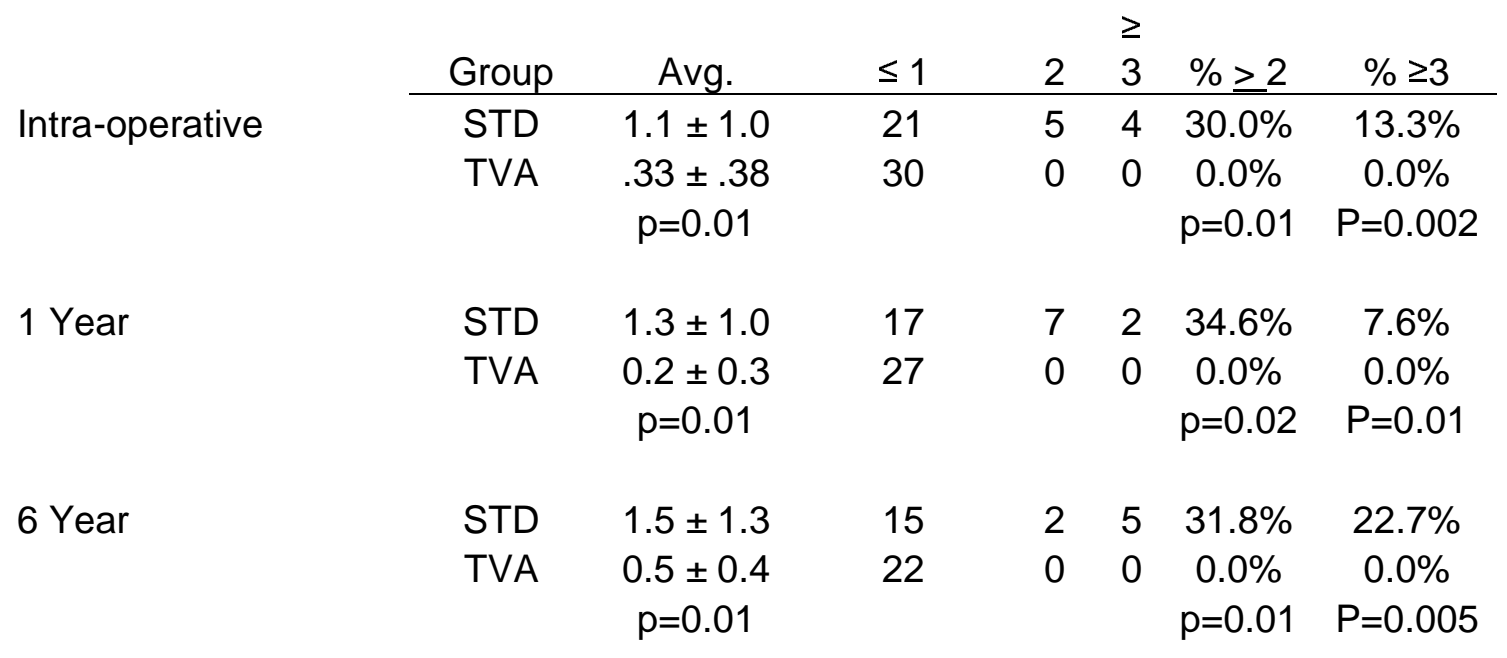

Jeevanandam, et al. Page 20 of 24 
Table 4 - Hemodynamic measurements

\begin{tabular}{lcccccc}
\multirow{4}{*}{1 Year } & Group & mSys & mPA & CVP & mPA-CVP & Cl \\
\cline { 2 - 7 } & STD & $82.1 \pm 10.7$ & $19.1 \pm 9.2$ & $6.5 \pm 4.3$ & $13.4 \pm 6.5$ & $3.6 \pm .7$ \\
& TVA & $87.3 \pm 9.8$ & $20.4 \pm 9.1$ & $6.9 \pm 4.9$ & $14.3 \pm 6.32$ & $3.3 \pm .6$ \\
& & & ns & ns & ns & ns
\end{tabular}

6 year

$84.2 \pm 12.1$

STD

TVA $\quad 82.6 \pm 11.8$

$23 \pm 5.2$

$4.4 \pm 3.2$

$15 \pm 3.2$

$14.5 \pm 4.2$

$2.8 \pm .5$

ns

ns

ns

$2.9 \pm .5$

ns

ns

$\mathrm{mSys}(\mathrm{mmHg})$ : mean systemic blood pressure

$\mathrm{mPA}(\mathrm{mmHg})$ : mean pulmonary artery pressure

CVP $(\mathrm{mmHg})$ : central venous pressure

$\mathrm{Cl}(\mathrm{L} / \mathrm{min} / \mathrm{m} 2)$ : cardiac index

Jeevanandam, et al. Page 21 of 24 
Table 5 - Renal function following heart transplantation

\begin{tabular}{ccccc} 
& Group & BUN & Crt & dCrt \\
\cline { 2 - 5 } Pre-operative & STD & $22.3 \pm 11.5$ & $1.02 \pm .3$ & \\
& TVA & $26.8 \pm 15.6$ & $1.34 \pm .7$ \\
& & ns & $\mathrm{p}=0.058$
\end{tabular}

1 Year

$\begin{array}{cccc}\text { STD } & 42.5 \pm 16.5 & 2.3 \pm 1.3 & 1.3 \pm .9 \\ \text { TVA } & 37.7 \pm 15 & 2.2 \pm 1 & 1.0 \pm .9 \\ & \text { ns } & \text { ns } & p=0.061\end{array}$

6 Year

$\begin{array}{cccc}\text { STD } & 41.0 \pm 14.4 & 2.9 \pm 2.0 & 2.0 \pm 2.1 \\ \text { TVA } & 32.1 \pm 12.1 & 1.8 \pm 0.7 & 0.7 \pm .8 \\ & n s & P=0.01 & \mathrm{p}=0.005\end{array}$

$\mathrm{BUN}(\mathrm{mg} / \mathrm{dl})$ : blood urea nitrogen Crt $(\mathrm{mg} / \mathrm{dll})$ : serum creatinine $\mathrm{dCrt:}$ difference in creatinine from baseline 
Table 7 - Complications and mortality following heart transplantation

\begin{tabular}{|c|c|c|c|c|}
\hline Group & $\begin{array}{l}\text { Intra-operative } \\
\text { complications }\end{array}$ & $\begin{array}{c}\text { Mortality } \\
1 \text { year }\end{array}$ & $\begin{array}{l}\text { Mortality } \\
6 \text { year }\end{array}$ & $\begin{array}{c}\text { Cardiac } \\
\text { deaths }\end{array}$ \\
\hline$A$ & $\begin{array}{l}\text { Bleeding } 3 \\
\text { Pul HTN/RHF } 5 \\
\text { CVA } 1 \\
\text { Pulm hem } 1 \\
\text { Donor dys } 2\end{array}$ & $\begin{array}{l}\text { RHF } 3 \\
\text { rejection } 1\end{array}$ & $\begin{array}{l}\text { CHF } 3 \\
\text { cancer } 1\end{array}$ & 7 \\
\hline B & $\begin{array}{l}\text { Bleeding } 3 \\
\text { Pul HTN/RHF } 4 \\
\text { Donor dys } 3\end{array}$ & $\begin{array}{l}1 \text { rejection } \\
1 \text { sepsis } \\
1 \text { pneumonia } \\
\text { ns }\end{array}$ & $\begin{array}{l}\text { CHF } 2 \\
\text { Perforated } \\
\text { bowel } 2 \\
\begin{array}{c}\text { Pneumonia } 1 \\
\text { ns }\end{array}\end{array}$ & $P=0.03$ \\
\hline
\end{tabular}

Pul HTN - pulmonary hypertension

$\mathrm{RHF}$ - right heart failure

CVA - cerebral vascular accident

Pulm hem - pulmonary hemorrhage

Donor dys - biventricular donor

dysfunction

CHF - allograft failure

Jeevanandam, et al. Page 23 of 24 
Figure 1: Survival in patients with tricuspid regurgitation $\geq 2+$

Figure 2: Linear regression showing correlation between tricuspid regurgitation and elevation in serum creatinine

Figure 3: Survival in patients with elevation is serum creatinine $\geq 2.5 \mathrm{mg} / \mathrm{dl}$

Jeevanandam, et al. Page 24 of 24 
1 $\quad$ Figure. 1 Survival in patients with $\geq 2+$ Tricuspid

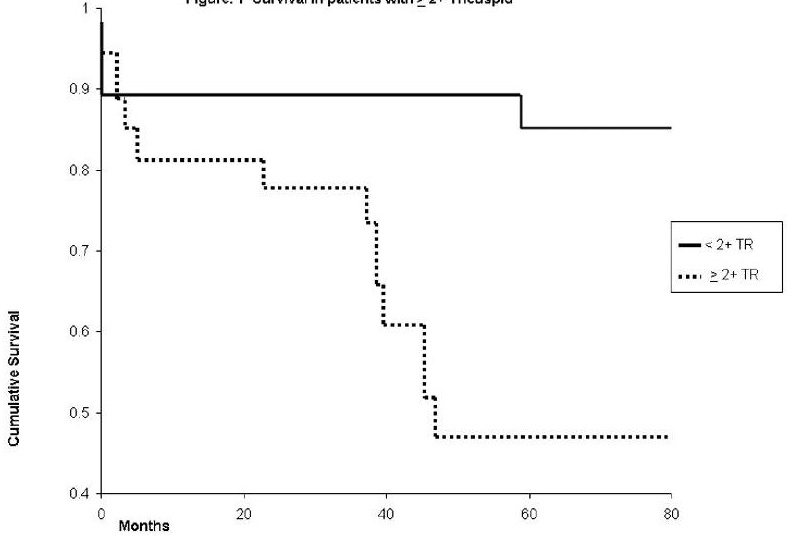


Figure 2. Linear regression analysis between tricuspid regurgitation and elevation in creatinine

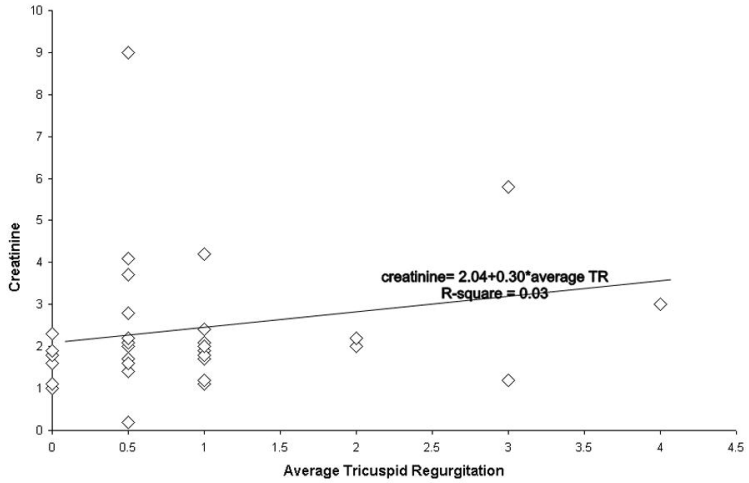


Survival curve for patients with Creatinine $\geq 2.5$

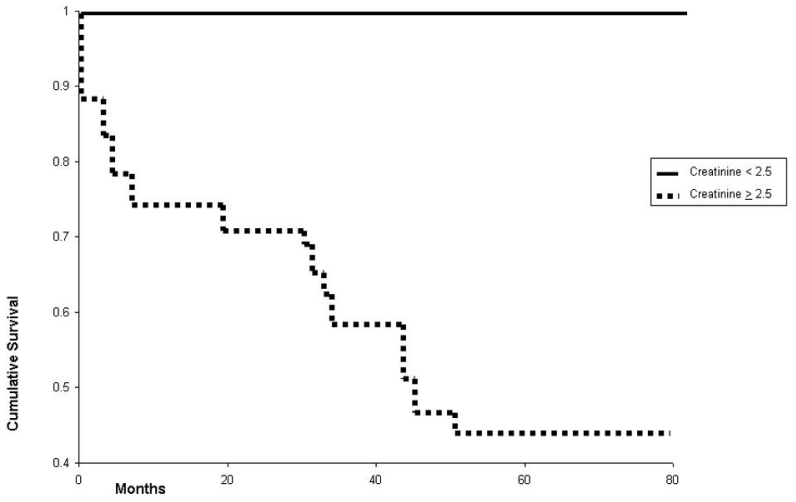

